DOI: $\mathrm{xxx} / \mathrm{xxxx}$

\title{
PROCEEDINGS
}

\section{Precession of triaxially deformed neutron stars $\|^{\dagger}$}

\author{
Yong Gao ${ }^{* 1,2}$ | Lijing Shao ${ }^{2,3}$
}

\author{
${ }^{1}$ Department of Astronomy, School of \\ Physics, Peking University, Beijing 100871, \\ China \\ ${ }^{2}$ Kavli Institute for Astronomy and \\ Astrophysics, Peking University, Beijing \\ 100871, China \\ ${ }^{3}$ National Astronomical Observatories, \\ Chinese Academy of Sciences, Beijing \\ 100012, China
}

\section{Correspondence}

*Email: gaoyong.physics@pku.edu.cn

\section{Funding Information}

National Natural Science Foundation of China, 11975027, 11991053,

11721303. China Association for Science and Technology, 2018QNRC001. Max Planck Society, Max Planck Partner Group. Chinese Academy of Sciences, XDB23010200.

\begin{abstract}
A deformed neutron star (NS) will precess if the instantaneous spin axis and the angular momentum are not aligned. Such a precession can produce continuous gravitational waves (GWs) and modulate electromagnetic pulse signals of pulsars. In this contribution we extend our previous work in a more convenient parameterization. We treat NSs as rigid triaxial bodies and give analytical solutions for angular velocities and Euler angles. We summarize the general GW waveforms from freely precessing triaxial NSs and use Taylor expansions to obtain waveforms with a small wobble angle. For pulsar signals, we adopt a simple cone model to study the timing residuals and pulse profile modulations. In reality, the electromagnetic torque acts on pulsars and affects the precession behavior. Thereof, as an additional extension to our previous work, we consider a vacuum torque and display an illustrative example for the residuals of body-frame angular velocities. Detailed investigations concerning continuous GWs and modulated pulsar signals from forced precession of triaixal NSs will be given in future studies.
\end{abstract}

\section{KEYWORDS:}

gravitational waves - pulsars: general - methods: analytical

\section{1 | INTRODUCTION}

A key challenge of modern astrophysics is to obtain the information on the internal structures of neutron stars (NSs). The precession of deformed NSs can modulate the precise pulsar timing signals (Jones \& Andersson, 2001, Link \& Epstein, 2001) and produce continuous gravitational waves (GWs; Jones \& Andersson, 2002; Zimmermann, 1980), which provides a means to probe the structures of NSs.

In this short contribution, we describe the dynamics of freely-precessing triaxial NSs in Sec. 2. The continuous GWs, electromagnetic timing residuals, and pulse-width modulations are studied in Secs. 3 and 4 As the electromagnetic torque acts on the pulsars in reality, we discuss the vacuum electromagnetic torque and give an illustrative example of the motions in Sec. 5. Finally, we summarize our work in Sec.6.

'Poster presentation at the 9th International Workshop on Astronomy and Relativistic Astrophysics, 6-12 September, 2020.

${ }^{0}$ Abbreviations: NS, neutron star; GWs, gravitational waves

\section{2 | FREE PRECESSION OF TRIAXIAL NSS}

We treat NSs as triaxial rigid bodies and ignore the complex internal dissipation that may exist. In the body frame corotating with the NS, the equation of motion takes the following form (Landau \& Lifshitz, 1960)

$$
\dot{\boldsymbol{L}}+\boldsymbol{\omega} \times \boldsymbol{L}=0,
$$

where the dot denotes the derivative with respect to time $t, \omega$ is the angular velocity, and $\boldsymbol{L}=\boldsymbol{I} \cdot \boldsymbol{\omega}$ is the angular momentum with $\boldsymbol{I}$ represents the moment of inertia tensor. Let $\widehat{\boldsymbol{e}}_{1}, \widehat{\boldsymbol{e}}_{2}$, and $\widehat{\boldsymbol{e}}_{3}$ denote the three unit eigenvectors along principal axes of $\boldsymbol{I}$ with corresponding eigenvalues $I_{1}, I_{2}$, and $I_{3}$. Then angular velocity is expressed as $\boldsymbol{\omega}=\omega_{1} \widehat{\boldsymbol{e}}_{1}+\omega_{2} \widehat{\boldsymbol{e}}_{2}+\omega_{3} \widehat{\boldsymbol{e}}_{3}$. For simplicity, at $t=0$ we set $\omega_{1}=a, \omega_{2}=0, \omega_{3}=b$, and the magnitude of the angular velocity $\omega=\omega_{0}$.

To describe the precession of NSs, we define

$$
\epsilon \equiv \frac{I_{3}-I_{1}}{I_{1}}, \quad \delta \equiv \frac{I_{2}-I_{1}}{I_{3}-I_{2}}, \quad \theta_{0} \equiv \arctan \frac{I_{1} a}{I_{3} b},
$$


where $\epsilon$ is the oblateness, $\delta$ is the non-axisymmetry, and $\theta_{0}$ is the initial wobble angle between $\boldsymbol{L}$ and $\hat{\boldsymbol{e}}_{3}$. To give an estimation of $\epsilon$, we consider two main causes of deformations: elasticity in the crust and the internal magnetic field. A small fraction of rotational bulge can misalign with the instantaneous rotation axis due to the elastic stress in the crystallized crust of NSs (Cutler, Ushomirsky, \& Link, 2003, Melatos, 2000). For a NS with mass $M$, radius $R$ and a constant shear modulus $\mu$ in the crust, the oblateness from elastic deformation is (Baym \& Pines, 1971, Gao et al. 2020)

$$
\epsilon_{\text {ela }} \simeq 4.9 \times 10^{-8}\left(\frac{\omega_{0}}{2 \pi \times 100 \mathrm{~Hz}}\right)^{2} \mu_{30} R_{6}^{7} M_{1.4}^{-3},
$$

where $M_{1.4}, R_{6}$, and $\mu_{30}$ represent $M /\left(1.4 M_{\odot}\right), R /\left(10^{6} \mathrm{~cm}\right)$, and $\mu /\left(10^{30} \mathrm{erg} \mathrm{cm}^{-3}\right)$ respectively. While the oblateness caused by internal magnetic field can be roughly estimated as (Lasky \& Melatos, 2013; Zanazzi \& Lai, 2015)

$$
\epsilon_{\mathrm{mag}} \simeq \frac{B^{2} R^{4}}{G M^{2}}=2 \times 10^{-12} B_{12}^{2} R_{6}^{4} M_{1.4}^{-2},
$$

where $B$ is the internal magnetic field and $B_{12}$ is $B /\left(10^{12} \mathrm{G}\right)$. In general, the combination of the elastic field in the crust and the internal magnetic field deform the NS into a triaxial shape and the non-axisymmetry can be any positive value. The biaxial case is a good approximation only if one of the deformation causes can be ignored compared to the other $\left(\epsilon_{\text {ela }} \ll \epsilon_{\text {mag }}\right.$ or $\left.\epsilon_{\text {ela }} \ll \epsilon_{\text {mag }}\right)$, and the deformation is symmetric about a specific axis instead of the rotational axis (Melatos 2000).

Eq. (1) can be solved analytically. With a more convenient dimensionless parameterization than that in Gao et al. (2020), we obtain the time evolution of $u_{i} \equiv \omega_{i} / \omega_{0}(i=1,2,3)$ in terms of elliptic functions $\mathrm{cn}$, sn, and dn (Landau \& Lifshitz, 1960: Zimmermann 1980). This new parameterization is presumed to have a more stable numerical behavior, in particular when a torque term is added (see Sec. 5). When $L^{2}>2 E I_{2}$, the solution takes the following form

$$
\begin{aligned}
& u_{1}(t)=u_{10} \operatorname{cn}\left(\omega_{\mathrm{p}} t, m\right), \\
& u_{2}(t)=u_{10}\left[\frac{(1+\delta)^{2}}{1+\delta+\epsilon \delta}\right]^{1 / 2} \operatorname{sn}\left(\omega_{\mathrm{p}} t, m\right), \\
& u_{3}(t)=u_{30} \operatorname{dn}\left(\omega_{\mathrm{p}} t, m\right),
\end{aligned}
$$

for $u_{1}=u_{10} \equiv a / \omega_{0}, u_{2}=0$, and $u_{3}=u_{30} \equiv b / \omega_{0}$ at $t=0$, where the parameters $\omega_{\mathrm{p}}$ and $m$ are

$$
\begin{aligned}
& \omega_{\mathrm{p}}=u_{30} \omega_{0} \epsilon(1+\delta+\delta \epsilon)^{-1 / 2}, \\
& m=\delta(1+\epsilon) \tan ^{2} \theta_{0} .
\end{aligned}
$$

The dimensionless variables $u_{i}$ are periodic with a period

$$
T=\frac{4 K(m)(1+\delta+\delta \epsilon)^{1 / 2}}{u_{30} \omega_{0} \epsilon},
$$

where $K(m)$ is the complete elliptic integral of the first kind (Landau \& Lifshitz 1960). In the biaxial case $(\delta=0$ or $\infty$ ), the elliptic integral $K(m)$ becomes $\pi / 2$, leading to $T=2 \pi / \omega_{\mathrm{p}}$.
The motion of the NS in the inertial frame can be described by three Euler angles: $\phi, \theta$, and $\psi$. We take the unit basis vectors of the coordinate system in the inertial frame as $\hat{\boldsymbol{e}}_{\mathrm{X}}, \widehat{\boldsymbol{e}}_{\mathrm{Y}}$, and $\hat{\boldsymbol{e}}_{\mathrm{Z}}$. We let $\widehat{\boldsymbol{e}}_{\mathrm{Z}}$ parallel to $\boldsymbol{L}$ and define $\hat{\boldsymbol{N}}=\widehat{\boldsymbol{e}}_{\mathrm{Z}} \times \widehat{\boldsymbol{e}}_{3}$. Then, the Euler angles satisfy

$$
\cos \phi=\hat{\boldsymbol{e}}_{\mathrm{X}} \cdot \hat{\boldsymbol{N}}, \quad \cos \theta=\widehat{\boldsymbol{e}}_{3} \cdot \widehat{\boldsymbol{e}}_{\mathrm{Z}}, \quad \cos \psi=\widehat{\boldsymbol{e}}_{1} \cdot \widehat{\boldsymbol{N}} .
$$

The angles $\theta$ and $\psi$ are both periodic with period $T$ (Landau \& Lifshitz, 1960)

$$
\begin{aligned}
& \cos \theta=\cos \theta_{0} \operatorname{dn}\left(\omega_{\mathrm{p}} t, m\right), \\
& \tan \psi=\left[\frac{1}{1+\delta+\delta \epsilon}\right]^{1 / 2} \frac{\operatorname{cn}\left(\omega_{\mathrm{p}} t, m\right)}{\operatorname{sn}\left(\omega_{\mathrm{p}} t, m\right)} .
\end{aligned}
$$

The angle $\phi$ equals to $\phi_{1}+\phi_{2}$, where (Landau \& Lifshitz, 1960)

$$
\begin{aligned}
\exp \left[2 \mathrm{i} \phi_{1}(t)\right] & =\frac{\vartheta_{4}\left(\frac{2 \pi}{T} t+\mathrm{i} \pi \alpha, q\right)}{\vartheta_{4}\left(\frac{2 \pi}{T} t-\mathrm{i} \pi \alpha, q\right)}, \\
\phi_{2}=\frac{2 \pi}{T_{1}} t & =\left(\frac{(1+\epsilon) u_{30} \omega_{0}}{\cos \theta_{0}}+\frac{2 \pi \mathrm{i}}{T} \frac{\vartheta_{4}^{\prime}(\mathrm{i} \pi \alpha, q)}{\vartheta_{4}(\mathrm{i} \pi \alpha, q)}\right) t .
\end{aligned}
$$

Here $\vartheta_{4}$ is the fourth Jacobian theta functions with the nome $q=\exp [-\pi K(1-m) / K(m)]$, and $\alpha$ can be obtained via $\operatorname{sn}[2 \mathrm{i} \alpha K(m)]=\mathrm{i} \cot \theta_{0}$. Once the values of $\epsilon, \delta, \theta_{0}$, and $\omega_{0}$ are given, one gets the time evolution of the NS at any time from Eqs. (5-15).

\section{3 | CONTINUOUS GWS}

Free precession can be manifested in continuous GWs. If the precessing triaxial NS rotates rapidly, the timing varying mass quadrupole generates continuous GWs lie in the kilohertz $(\mathrm{kHz})$ band, which is to be observed by ground-based GW detectors like LIGO, Virgo, and KAGRA.

The general GW waveforms for freely precessing triaxial NSs are (Van Den Broeck, 2005, Zimmermann, 1980)

$$
\begin{aligned}
h_{+}= & -\frac{G}{r c^{4}}\left[\left(\mathcal{R}_{2 i} \cos \imath+\mathcal{R}_{3 i} \sin \imath\right)\left(\mathcal{R}_{2 j} \cos \imath+\mathcal{R}_{3 j} \sin \imath\right)\right. \\
& \left.\quad-\mathcal{R}_{1 i} \mathcal{R}_{1 j}\right] A_{i j}, \\
h_{\times}= & -\frac{2 G}{r c^{4}}\left(\mathcal{R}_{2 i} \cos \imath+\mathcal{R}_{3 i} \sin \imath\right) \mathcal{R}_{1 j} A_{i j},
\end{aligned}
$$

where $h_{+}$and $h_{\times}$are "+" and "X" polarized GWs, $r$ is the luminosity distance to the NS, $l$ is the inclination angle between the line of sight and $\hat{\boldsymbol{e}}_{\mathrm{Z}}$. Here $\mathcal{R}_{i j}$ is the rotation matrix, which can be represented by $\phi, \theta$, and $\psi$. The tensor $A_{i j}$ is a function of the moment of inertia tensor $\boldsymbol{I}$ and the angular velocities $\omega_{i}$ (see Eq. (21) in Zimmermann (1980)). In the frequency domain, the emission of GWs mainly occurs at frequencies

$$
f_{\mathrm{r}}+(2 n+1) f_{\mathrm{p}}, \quad 2 f_{\mathrm{r}}+2 n f_{\mathrm{p}},
$$

where $f_{\mathrm{p}}=1 / T$ is the free precession frequency, $f_{\mathrm{r}}$ equals to $1 / T_{1}-1 / T$, and $n$ is an integer. 
In the case of small oblatenesses, small wobble angles and small non-axisymmetries, one can use Taylor expansions of $\theta$ and $\delta$ to obtain the waveforms. The first order contributions occurs at $f_{\mathrm{r}}+f_{\mathrm{p}}$ and $2 f_{\mathrm{r}}$ (Van Den Broeck. 2005), with amplitudes

$$
\begin{aligned}
& A_{\times}^{1}=2 \times 10^{-28} \theta_{0} \sin \imath\left(\frac{\epsilon}{10^{-8}}\right)\left(\frac{f_{\mathrm{r}}}{100 \mathrm{~Hz}}\right)^{2}\left(\frac{1 \mathrm{kpc}}{r}\right), \\
& A_{\times}^{2}=4 \times 10^{-28} \delta \cos \imath\left(\frac{\epsilon}{10^{-8}}\right)\left(\frac{f_{\mathrm{r}}}{100 \mathrm{~Hz}}\right)^{2}\left(\frac{1 \mathrm{kpc}}{r}\right),
\end{aligned}
$$

for " $\times$ " polarized GWs. The amplitudes for " + " polarized GWs can be obtained similarly but with a different dependence on the inclination angle $t$. The second order lines are too weak (Gao et al. 2020) for current observational interests and we do not discuss them here.

\section{4 | MODULATIONS IN PULSARS}

For pulsars, the emission beam will rotates around the principal axis $\widehat{\boldsymbol{e}}_{3}$ during free precession and one can possibly observe timing residuals and pulse profile modulations with radio/Xray telescopes (Ashton, Jones, \& Prix, 2017, Gao et al., 2020, Jones \& Andersson, 2001, Link \& Epstein, 2001).

To study the timing residuals, we assume that the emission is along the magnetic dipole $\boldsymbol{m}$ and one can observe the pulsar signals once $\boldsymbol{m}$ sweeps through the plane defined by the line of sight and $\widehat{\boldsymbol{e}}_{\mathrm{Z}}$. We define

$$
\cos \chi=\widehat{\boldsymbol{m}} \cdot \widehat{\boldsymbol{e}}_{3}, \quad \cos \Phi=\widehat{\boldsymbol{e}}_{\mathrm{X}} \cdot \widehat{\boldsymbol{M}}, \quad \cos \Theta=\widehat{\boldsymbol{m}} \cdot \widehat{\boldsymbol{e}}_{\mathrm{Z}},
$$

where $\hat{\boldsymbol{m}}$ is $\boldsymbol{m} /|\boldsymbol{m}|, \chi$ is the angle between $\widehat{\boldsymbol{m}}$ and $\widehat{\boldsymbol{e}}_{3}, \widehat{\boldsymbol{M}}=$ $\hat{\boldsymbol{e}}_{\mathrm{Z}} \times\left(\hat{\boldsymbol{m}} \times \widehat{\boldsymbol{e}}_{\mathrm{Z}}\right)$ is the unit vector along the projection of $\boldsymbol{m}$ on the $\hat{e}_{X}-\hat{e}_{Y}$ plane, $\Phi$ and $\Theta$ are the azimuthal and the polar angles of $\hat{\boldsymbol{m}}$ in the inertial frame. The azimuthal angle $\Phi$ can be expressed as (Jones \& Andersson, 2001)

$$
\Phi=\phi-\frac{\pi}{2}+\arctan \left(\frac{\cos \psi \sin \chi}{\sin \theta \cos \chi-\sin \psi \sin \chi \cos \theta}\right),
$$

and the phase residual due to precession is

$$
\Delta \Phi=\Phi-\langle\Phi\rangle,
$$

which depends on the relative values of the wobble angle $\theta$ and the angle $\chi$ (see Eqs. (45) and (48) in Jones \& Andersson (2001)). In Eq. (23), " $\langle\cdot\rangle$ ” means the time averaged values. The mean spin period of the pulsar is $P_{0}=2 \pi /\langle\dot{\Phi}\rangle$. Thus, the timing residual of the spin period $P$ is

$$
\Delta P=\frac{2 \pi}{\dot{\Phi}}-\frac{2 \pi}{\langle\dot{\Phi}\rangle} \simeq-\frac{P_{0}^{2}}{2 \pi} \Delta \dot{\Phi} .
$$

To the second order expansions of $\theta_{0}$ and $\delta$ with a small wobble angle and a small non-axisymmetry, the period residual is (Gao et al. 2020)

$$
\begin{aligned}
\Delta P \approx & \frac{P_{0}^{2}}{2} f_{\mathrm{p}} \theta_{0}(2 \delta+1) \cot \chi \cos \left(2 \pi f_{\mathrm{p}} t\right) \\
& +\frac{P_{0}^{2}}{2} f_{\mathrm{p}} \theta_{0}^{2}\left(1+2 \cot ^{2} \chi\right) \cos \left(4 \pi f_{\mathrm{p}} t\right) .
\end{aligned}
$$

Note that the period derivative residual $\Delta \dot{P}$ can be obtained directly by taking the time derivative of $\Delta P$.

The polar angle $\Theta$,

$$
\cos \Theta=\sin \theta \sin \psi \sin \chi+\cos \theta \cos \chi,
$$

varies as a function of time. The line of sight cuts different region of the emission cone during free precession and one could observe pulse width modulations. Adopting a simple cone model, the pulse width $W$ reads (Gil, Gronkowski, \& Rudnicki, 1984, Lorimer \& Kramer, 2005)

$$
\sin ^{2}\left(\frac{W}{4}\right)=\frac{\sin ^{2}(\rho / 2)-\sin ^{2}(\beta / 2)}{\sin (\Theta+\beta) \sin \Theta},
$$

where $\rho$ is the angular radius of the emission cone, $\beta$ is the impact parameter corresponding to the closest approach between the magnetic dipole moment and the line of sight. Detailed results can be found in Gao et al. (2020). Pulse-width modulations can be used to infer the emission shape and pulsar radiation properties (Link \& Epstein, 2001).

\section{5 | FORCED PRECESSION DUE TO AN ELECTROMAGNETIC TORQUE}

In our previous work (Gao et al. 2020), we assume that the precession is free, namely without a torque. However, in reality the existence of electromagnetic torque will modulate the free precession and affect continuous GWs and pulsar signals. We take a vacuum electromagnetic torque as an example (Goldreich 1970; Jones \& Andersson, 2002, Zanazzi \& Lai, 2015)

$$
\boldsymbol{T}=\boldsymbol{T}_{1}+\boldsymbol{T}_{2}=\frac{2 \omega^{2}}{3 c^{3}}(\boldsymbol{\omega} \times \boldsymbol{m}) \times \boldsymbol{m}+\frac{3}{5 R c^{2}}(\boldsymbol{\omega} \cdot \boldsymbol{m})(\boldsymbol{\omega} \times \boldsymbol{m}),
$$

and ignore the complex magnetospheric processes Arzamasskiy, Philippov, \& Tchekhovskoy. 2015). The first term, $\boldsymbol{T}_{1}$, is the secular spin down torque, which has components both parallel and perpendicular to the angular momentum $\boldsymbol{L}$. The component parallel to $\boldsymbol{L}$ accounts for the usual spin down and the component perpendicular to $\boldsymbol{L}$ is responsible for the change of the wobble angles (Jones \& Andersson 2002). The second term, $\boldsymbol{T}_{2}$, is the anomalous torque, which originates from the moment of inertia of magnetic dipole field (Melatos 2000, Zanazzi \& Lai 2015). This torque is perpendicular to the angular velocity $\omega$ and does not decrease the energy or the angular momentum. However, it changes the spin period and the wobble angles for precessing NSs (Jones \& Andersson, 2002). 


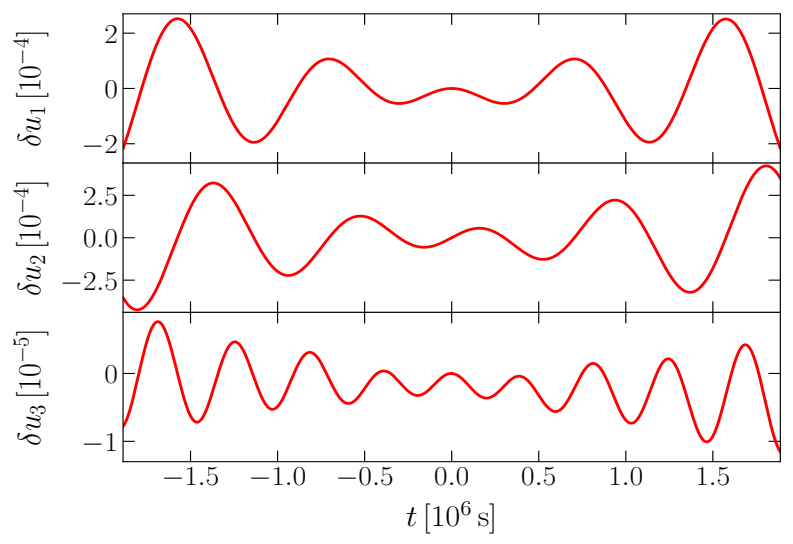

FIGURE 1 An illustrative example for the residuals $\delta u_{1}, \delta u_{2}$, and $\delta u_{3}$ due to electromagnetic torque $\boldsymbol{T}$. Here we take $\epsilon=$ $10^{-5}, \delta=1, \theta_{0}=3^{\circ}, \omega_{0}=1 \mathrm{rad} \mathrm{s}^{-1}$, and $\chi=89.5^{\circ}$. The time scales $\tau_{\mathrm{c}}$ and $\tau_{\mathrm{p}}$ are set to be $10^{12} \mathrm{~s}$ and $1.11 \times 10^{8} \mathrm{~s}$.

The Euler equation for the forced precession with $\boldsymbol{T}$ is (Arzamasskiy et al. 2015)

$$
\begin{aligned}
\dot{\boldsymbol{u}} & +\frac{1}{\tau_{\mathrm{p}}}\left(\frac{\delta}{1+\delta} \dot{u}_{2} \widehat{\boldsymbol{e}}_{2}+\dot{u}_{3} \widehat{\boldsymbol{e}}_{3}+\frac{\delta}{1+\delta} u_{2} \boldsymbol{u} \times \widehat{\boldsymbol{e}}_{2}+u_{3} \boldsymbol{u} \times \widehat{\boldsymbol{e}}_{3}\right) \\
= & \frac{u^{2}}{\tau_{\mathrm{c}}}[(\boldsymbol{u} \cdot \hat{\boldsymbol{m}}) \hat{\boldsymbol{m}}-\boldsymbol{u}]+\frac{1}{\tau_{a}}[(\boldsymbol{u} \cdot \hat{\boldsymbol{m}})(\boldsymbol{u} \times \hat{\boldsymbol{m}})] .
\end{aligned}
$$

The precession time scale $\tau_{\mathrm{p}}$, the secular spin down time scale $\tau_{\mathrm{c}}$ (corresponding to $\boldsymbol{T}_{1}$ ), and the time scale $\tau_{\mathrm{a}}$ (corresponding to $\boldsymbol{T}_{2}$ ) are, respectively,

$$
\tau_{\mathrm{p}}=1 / \epsilon \omega_{0}, \quad \tau_{\mathrm{c}}=3 c^{3} I_{1} / 2 m^{2} \omega_{0}^{2}, \quad \tau_{\mathrm{a}}=10 R \omega_{0} \tau_{\mathrm{c}} / 9 c,
$$

where the relation $\tau_{\mathrm{p}} \ll \tau_{\mathrm{a}} \ll \tau_{\mathrm{c}}$ is usually satisfied Arzamasskiy et al. 2015). We show an illustrative example in Fig. 1 for forced precession. One notices that $u_{i}$ oscillates during the forced precession, which is mainly caused by the torque $\boldsymbol{T}_{2}$. In this small initial wobble angle case, the angular velocity $\omega \simeq \omega_{0} u_{3}$ and $u_{3}$ decreases on a longer time scale caused by the spin down torque $\boldsymbol{T}_{1}$. Because of the relation $\tau_{\mathrm{p}} \ll \tau_{\mathrm{a}} \ll \tau_{\mathrm{c}}$, one can actually treat torques as perturbation on the free precession in multi-timescale analysis Arzamasskiy et al. 2015, Link \& Epstein 2001).

The continuous GWs and pulsar signals from forcedprecessing triaxial NSs have some new features and we plan to present them in detail in future studies.

\section{6 | SUMMARY}

We gave the analytical solutions of freely-precessing triaxial NSs and studied their characteristics in continuous GWs and pulsar signals. These results are ready to be used for future searches of precession. We also discussed the effects of the electromagnetic torque on the motions of precession, which deserve more studies in the future. From observations, precession will give us important information on the equation of state of NSs and related astrophysical properties (Gao et al. 2020).

\section{ACKNOWLEDGMENTS}

We are grateful to Rui Xu, Ling Sun, Chang Liu, and Ren-Xin $\mathrm{Xu}$ for discussions. This work was supported by the National Natural Science Foundation of China under Grant Nos. 11975027, 11991053, 11721303, the Young Elite Scientists Sponsorship Program by the China Association for Science and Technology under the Grant No. 2018QNRC001, and the Max Planck Society through the Max Planck Partner Group. It was partially supported by the Strategic Priority Research Program of the Chinese Academy of Sciences under the Grant No. XDB23010200, and the High-performance Computing Platform of Peking University.

\section{Conflict of interest}

The author declares no potential conflict of interests.

\section{REFERENCES}

Arzamasskiy, L., Philippov, A., \& Tchekhovskoy, A. 2015, MNRAS, $453,3540-3553$.

Ashton, G., Jones, D., \& Prix, R. 2017, MNRAS, 467, 164-178.

Baym, G., \& Pines, D. 1971, Ann. Phys., 66, 816-835.

Cutler, C., Ushomirsky, G., \& Link, B. 2003, ApJ, 588, 975.

Gao, Y., Shao, L., Xu, R., Sun, L., Liu, C., \& Xu, R.-X. 2020, MNRAS, 498, 1826-1838.

Gil, J., Gronkowski, P., \& Rudnicki, W. 1984, A\&A, 132, 312-316.

Goldreich, P. 1970, ApJL, 160, L11.

Jones, D. I., \& Andersson, N. 2001, MNRAS, 324, 811.

Jones, D. I., \& Andersson, N. 2002, MNRAS, 331, 203.

Landau, L. D., \& Lifshitz, E. M. 1960, Mechanics. Oxford.

Lasky, P. D., \& Melatos, A. 2013, Phys. Rev. D, 88, 103005.

Link, B., \& Epstein, R. I. 2001, ApJ, 556, 392.

Lorimer, D. R., \& Kramer, M. 2005, Handbook of Pulsar Astronomy. Cambridge, England: Cambridge University Press.

Melatos, A. 2000, MNRAS, 313, 217.

Van Den Broeck, C. 2005, Class. Quant. Grav., 22, 1825-1840.

Zanazzi, J., \& Lai, D. 2015, MNRAS, 451, 695-704.

Zimmermann, M. 1980, Phys. Rev. D, 21, 891-898. 